

\title{
Dielectric properties of betaine arsenate crystal near the phase transition
}

\author{
A. Hek, S. Dacko, Z. Czapla
}

\section{To cite this version:}

A. Hek, S. Dacko, Z. Czapla. Dielectric properties of betaine arsenate crystal near the phase transition. Journal of Physics and Chemistry of Solids, 2009, 69 (8), pp.1963. 10.1016/j.jpcs.2008.02.006 . hal00538005

\section{HAL Id: hal-00538005 \\ https://hal.science/hal-00538005}

Submitted on 20 Nov 2010

HAL is a multi-disciplinary open access archive for the deposit and dissemination of scientific research documents, whether they are published or not. The documents may come from teaching and research institutions in France or abroad, or from public or private research centers.
L'archive ouverte pluridisciplinaire HAL, est destinée au dépôt et à la diffusion de documents scientifiques de niveau recherche, publiés ou non, émanant des établissements d'enseignement et de recherche français ou étrangers, des laboratoires publics ou privés. 


\section{Author's Accepted Manuscript}

Dielectric properties of betaine arsenate crystal near the phase transition

A. Hek, S. Dacko, Z. Czapla

PII: $\quad$ S0022-3697(08)00048-6

DOI: $\quad$ doi:10.1016/j.jpcs.2008.02.006

Reference: $\quad$ PCS 5387

To appear in: Journal of Physics and

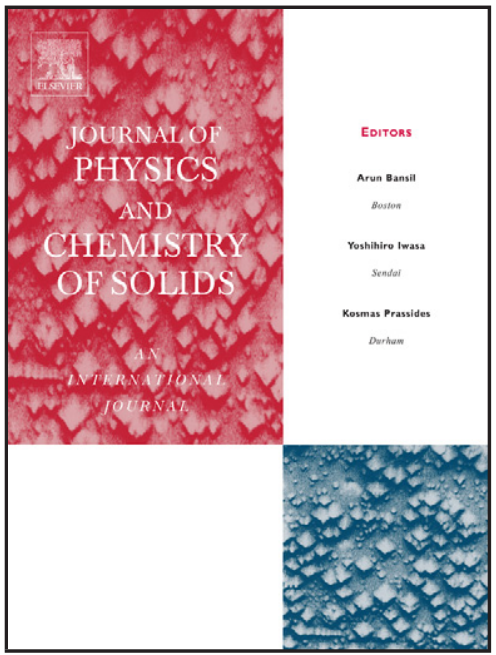

www.elsevier.com/locate/jpcs Chemistry of Solids

Received date: 9 June 2006

Revised date: $\quad 3$ August 2007

Accepted date: $\quad 11$ February 2008

Cite this article as: A. Hek, S. Dacko and Z. Czapla, Dielectric properties of betaine arsenate crystal near the phase transition, Journal of Physics and Chemistry of Solids (2008), doi:10.1016/j.jpcs.2008.02.006

This is a PDF file of an unedited manuscript that has been accepted for publication. As a service to our customers we are providing this early version of the manuscript. The manuscript will undergo copyediting, typesetting, and review of the resulting galley proof before it is published in its final citable form. Please note that during the production process errors may be discovered which could affect the content, and all legal disclaimers that apply to the journal pertain. 


\title{
Dielectric properties of Betaine Arsenate crystal near the phase transition
}

\author{
A. Hek ${ }^{\text {a* }}$, S.Dacko ${ }^{\mathrm{a}}$, and Z. Czapla ${ }^{\mathrm{a}}$
}

${ }^{a}$ Institute of Experimental Physics, Wrocaw University, pl. M. Borna 9, 50-204 Wrocaw, Poland

Dielectric measurements for single crystal of betaine arsenate $\left(\mathrm{CH}_{3}\right)_{3} \mathrm{NCH}_{2} \mathrm{COO}$. $\mathrm{H}_{3} \mathrm{AsO}_{4}$ connected with the ferroelectric phase transition at $119 \mathrm{~K}$ were performed. The temperature dependence of electric permittivity was measured at dc electric fields up to $700 \mathrm{kV} / \mathrm{m}$. The results show significant suppression of the dielectric constant by the application of dc field. Deviation from the classical behavior was observed. The electric permittivity was also measured in the paraelectric phase at constant temperature as a function of electric field intensity up to $700 \mathrm{kV} / \mathrm{m}$. The electric permittivity might be well described by the classical relation with additional term including contribution to permittivity coming from clusters. The fit parameters indicate that the polar-clusters carries polarization $P_{0}=0.7-4.910^{-3} \mathrm{Cm}^{2}$ with the clusters size of $L=12-20 \mathrm{~nm}$.

\section{Introduction}

Ferroelectric crystal of betaine arsenate $\left(\left(\mathrm{CH}_{3}\right)_{3} \mathrm{NCH}_{2} \mathrm{COO} \cdot \mathrm{H}_{3} \mathrm{AsO}_{4}\right.$ abbreviated as BA) has been known since 1982 [1]. According to this first paper the crystal undergoes two phase transitions, one at $T_{C 1}=411 \mathrm{~K}$ and another one at $T_{C 2}=119 \mathrm{~K}$. The first phase transition was identified as a para-ferroelastic phase transition, while the second was identified as a para-ferroelectric one $[2,3]$. The high frequency dielectric measurements and Raman spectroscopy indicate that the phase transition at $T_{C 2}$ appears to be of the order-disorder type $[4,5]$. The shape of the heat capacity curve measured near $T_{C 2}$ by adiabatic calorimeter [6] suggests that the phase transition is of the second order. The phase transition at $T_{C 1}$ seems to be of the first order [7]. The symmetry of the BA crystal above $411 K$ is $P b n m$ [8] and in the range of $119 K<T<411 K$ is $P 121 / n 1$ [2]. There are some evidence for a third phase transition occurring at the temperature about $110 \mathrm{~K}$ $[9,10]$. The latest X-Ray studies suggested the appearance of the third phase transition at the temperature $107 K$ and the space group P1n1 was proposed for temperature below $119 K$ [10]. Most of the experimental investigations of BA show deviations from what is considered as a typical ferroelectric behaviour. The pyroelectric coefficient along the [001] direction reveals two anomalies in the temperature range $110-123 K$ [11]. The hysteresis loops in BA exhibit striking S-shaped curves in the interval of a few $K$ above $T_{C 2}$ [1]. Using a model of weakly coupled one-dimensional Ising chains, the temperature dependence of dielectric constant in paraelectric phase was described as referred by Müser [12]. The same authors observed a cross-over of effective critical exponent $\gamma$ to a value

\footnotetext{
${ }^{*}$ This work was supported by the University of Wrocaw grant 2568/W/IFD
} 
typical for short-range forces in paraelectric phase [13]. Recent measurements also show that in BA exists short range interaction above $119 K[5,10]$, which are called clusters.

The aim of our studies was to measure and describe dielectric properties of BA under influence of electric field. The description was aimed to check the possibility to use dielectric state equation with two or three terms and with additional term coming from clusters to get the best fitting of parameters, which describe phase transition.

\section{Experimental}

Polycrystals of BA were obtained from water solution of stoichiometric quantities of arsenic acid and betaine. Single crystals BA were grown from saturated water solution of dissolved polycrystals of the substance by slow evaporation method at constant temperature of $302 K$.

The c-plates approximately $0.7 \mathrm{~mm}$ in thickness were cut out from the crystal. Silver paste films were used as electrodes. The sample was placed in a nitrogen gas exchange chamber of a cryostat and it was mechanically free. The sample capacitance was measured using a precision LCR-meter HP 4284A with a measured field of $1 \mathrm{~V} / \mathrm{cm}$ in amplitude $1.2 \mathrm{kHz}$, allowing us to calculate the electric permittivity. The electric permittivity as a function of temperature was measured on a cooling run with a rate of $0.5 \mathrm{~K} / \mathrm{min}$ with different values of dc electric field from 0 to $700 \mathrm{kV} / \mathrm{m}$. Applying an electric field of amplitude $700 \mathrm{kV} / \mathrm{m}$ and the frequency $10^{-3} \mathrm{~Hz}$, the field dependence of the electric permittivity at constant temperatures was measured. The temperature in the chamber was controlled with an accuracy not worse than $5 \cdot 10^{-3} \mathrm{~K}$.

\section{Results and Discussion}

The dielectric constant and its reciprocal value are presented as a function of temperature in figure 1,2. In the paraelectric phase, the Curie-Weiss law :

$$
\varepsilon^{\prime}=\frac{C_{0}}{T-T_{0}}
$$

where $C_{0}$ and $T_{0}$ are Curie-Weiss constant and temperature, respectively. Equation (1) is well fulfilled in the temperature range of 120-150K (see dotted line in the figure 2) with $C_{01}=3.9 \pm 0.01 \cdot 10^{4} \mathrm{~K}$. However, this fit gives Curie-Weiss temperature different than the one in which maximum of dielectric constant appears. Another fit was done in the temperature range of $154-190 K$ (see solid line in the figure 2) and gave practically the same Curie-Weiss temperature as the temperature of the maximum of dielectric constant. The Curie-Weiss constant is equal to $C_{02}=3.6 \pm 0.01 \cdot 10^{4} \mathrm{~K}$. However, below temperature about $154 K$ experimental data show deviation from this fit. 


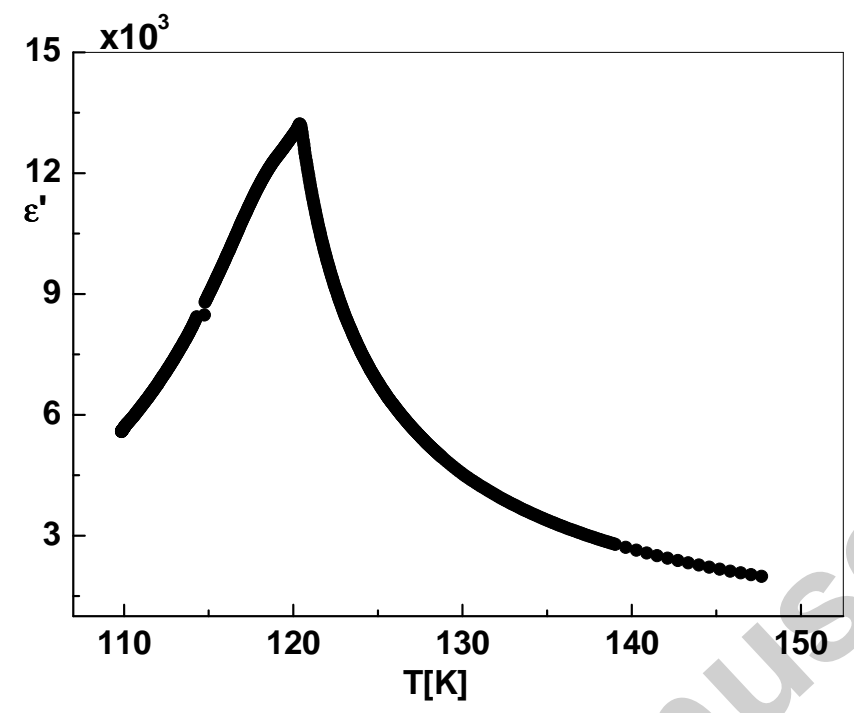

Figure 1. Temperature dependence of dielectric constant measured in c-axis direction.

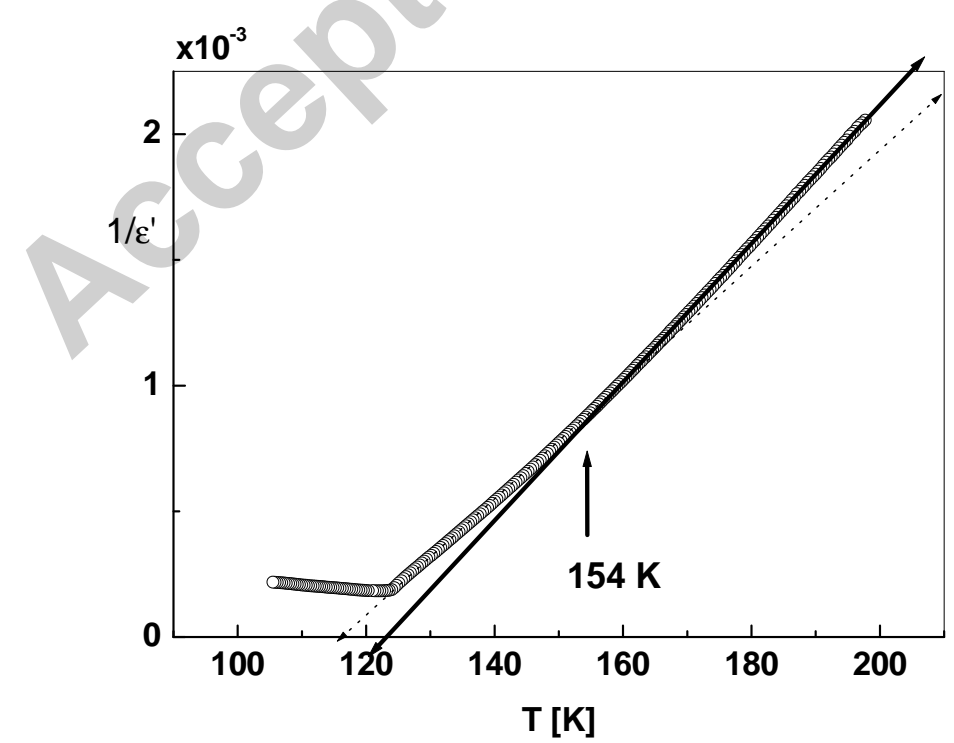

Figure 2. Temperature dependence of the reciprocal value of the dielectric constant. 


\subsection{Temperature dependence of electric permittivity in the paraelectric phase}

Figure 3 shows temperature variations of the dielectric constant for various dc electric fields. As it can be seen the maximum value of $\varepsilon^{\prime}$ decreases with intensity of applied electric field and the position of maximum shifts to higher temperatures. It looks like classical behaviour.

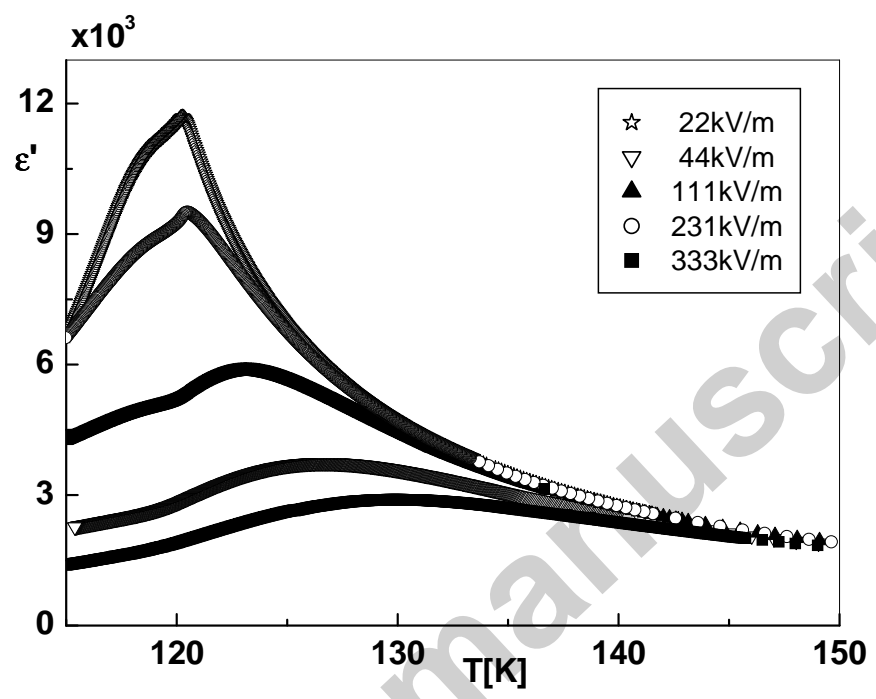

Figure 3. Temperature variation of the dielectric constant at various dc electric field.

However, when we take equation for maximum value of permittivity obtained from Landau theory [14]:

$\left(\varepsilon_{\max }\right)^{-1}=\frac{3}{2^{1 / 3}} B^{1 / 3} E^{1 / a}$

where $B$ is nonlinear coefficient in dielectric state equation, we do not get classical value of the exponent $a=1.5$. The experimental data are well described with the value of the exponent $a=1.66$. The results are presented in figure 4 . 
Dielectric properties of Betaine Arsenate

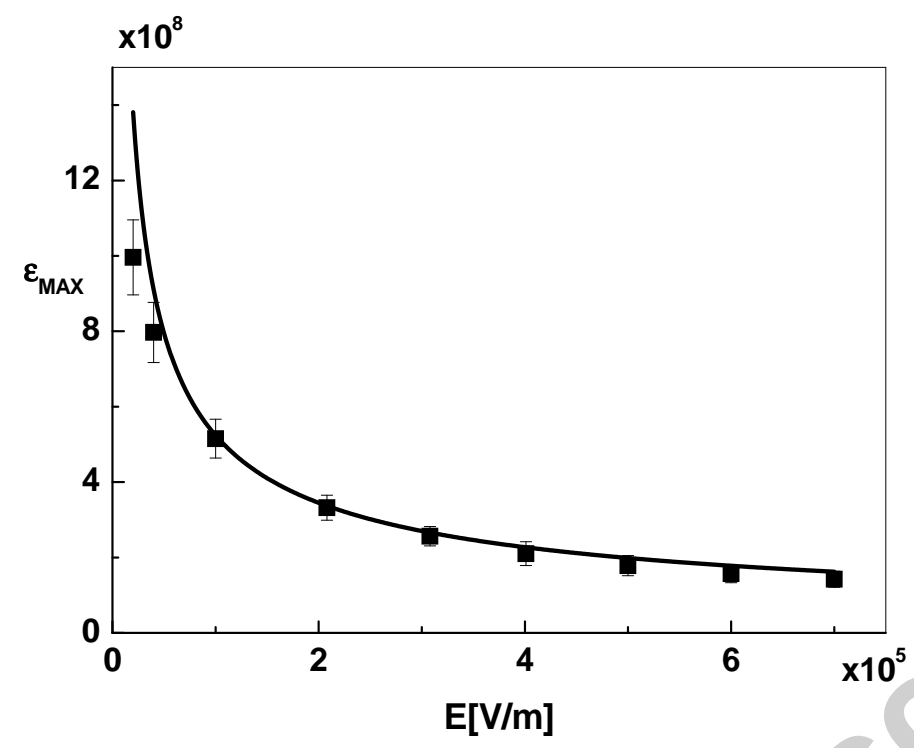

Figure 4. Maximum value of electric permittivity versus dc electric field for different temperatures.

\subsection{Field dependence of permittivity at constant temperatures in the para- electric phase}

The further study was focused on the dc electric field dependence of dielectric constant at constant temperature in the paraelectric phase. The measurements were done in the range of temperature $\left(T_{C 2}, T_{C 2}+30 K\right)$. In order to describe experimental data we started with the well-known classical dielectric state equation in a form:

$E=A P+B P^{3}+C P^{5}$,

where $A=A_{0}\left(T-T_{C}\right)$ is linear coefficient and $B, C$ are nonlinear one, which are assumed to be temperature independent. $P$ is an induced polarization.

Neglecting the last term of equation (3) and using relation:

$\left(\varepsilon_{0} \varepsilon^{\prime}\right)^{-1}=\frac{\partial E}{\partial P}$

one can rewrite (4) in the form:

$\varepsilon^{\prime}=1 /\left(\varepsilon_{0}\left(\mathrm{~A}+3 \mathrm{BP}^{2}\right)\right)$,

where the explicit relation between $P$ and $E$

$P=\left[\frac{E}{2 B}+\sqrt{\left(\frac{A}{3 B}\right)^{3}+\left(\frac{E}{2 B}\right)^{2}}\right]^{1 / 3}-\left[-\frac{E}{2 B}+{\sqrt{\left(\frac{A}{3 B}\right)^{3}+\left(\frac{E}{2 B}\right)^{2}}}^{1 / 3}\right.$

was obtained analytically from equation (3) with only two first terms.

On the other hand the field dependence of dielectric constant in the paraelectric state can be expressed as $[15,16]$

$\varepsilon^{\prime}=\frac{1}{\varepsilon_{0} A}-\frac{3 B}{\varepsilon_{0} A^{4}} E^{2}+\frac{15 B^{2}}{\varepsilon_{0} A^{7}} E^{4}$ 
This equation is commonly used to describe the behaviour of dielectric constant in dc field at constant temperature. However, in this work, fits of $\varepsilon^{\prime}$ vs. $E$ involving even powers of $E^{4}$ are insufficient to explain experimental data as it can be seen in figure 5 .

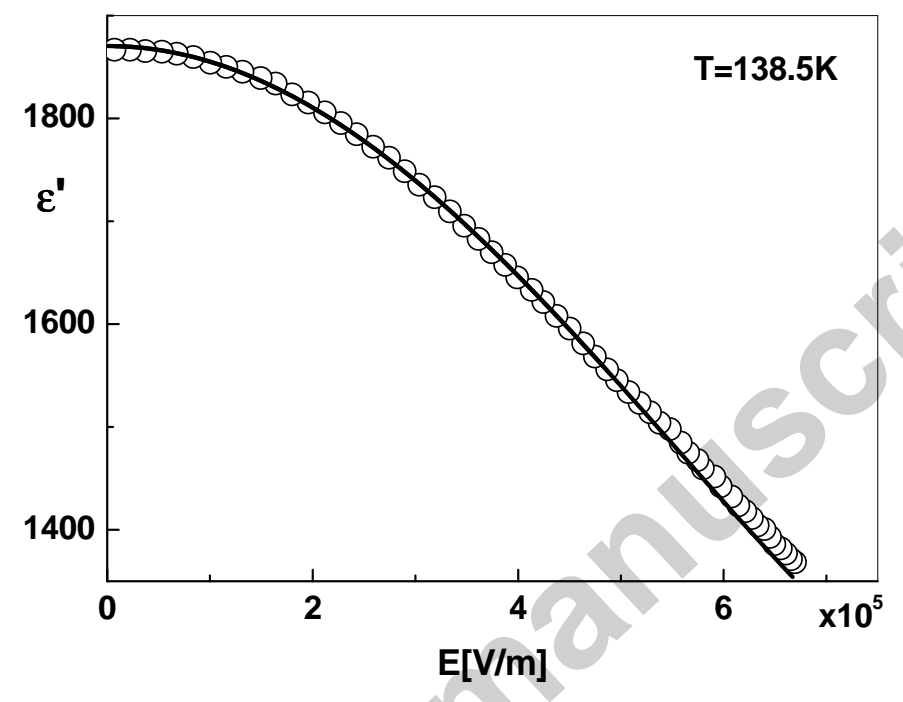

Figure 5. Field dependence of the dielectric constant and its best fit to the equation (7) (solid line).

Therefore, in next step the fits were done according to equation (5) and (6) as it was propose for some systems by Dec et al. [17]. Plot in figure 6 (see the dotted line) shows that fitting to equation (5) is in better agreement with data for temperature higher than $131 K$. From these fits the parameters $A$ and $B$ were obtained in the temperature range $132-152 K$. However, below $131 K$ fitting curves are in disagreement with experimental data as it can be seen in figure 7 .

Additionally, to explain experimental data we take into account another term $\left(E^{6}\right)$ in equation (7). We performed fitting with extended equation and result for $T=138.5 \mathrm{~K}$ is shown in figure 6 (see the solid line). Plots in the figure 8 show temperature dependence of fitting parameters $A$ and $B$. One might notice that in this case parameters $A$ and $B$ were in quite good agreement with the results from the equation (4). Nevertheless at the temperature below $126 K$, adding next term was insufficient to explain the semi-bell shape curves (as an example see figure 9).

Following results from $[5,10]$ we considered the appearance of clusters and according to method proposed by Bianchi et al. [18,19] and used by others authors [20], we added a cluster term to equation (5). By means of Langevin-type approach[21], the total polarization of a cluster system is expressed as

$P=P_{0} \tanh \left(\frac{P_{0} L^{3} E}{2 k_{B} T}\right)$. 


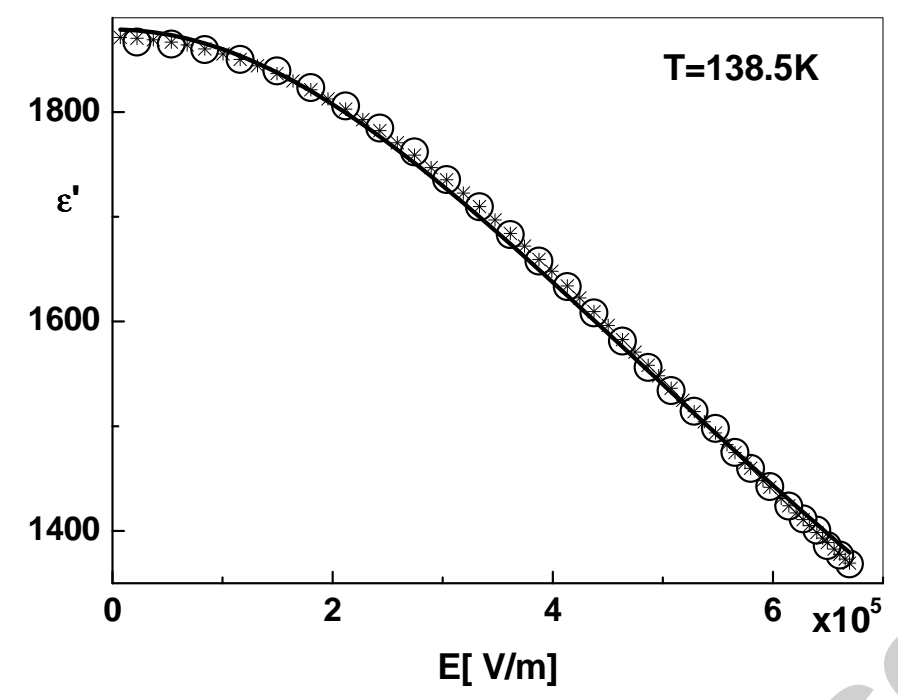

Figure 6. Field dependence of the dielectric constant and its best fit:

- solid line according to the equation (5)

- stars according to the equation (7) with additional term $E^{6}$.

here $P_{0}$ denotes effective polarization of clusters, $L$ the size of cluster, $k_{B}$ the Boltzmann's constant, and $E$ electric field. Therefore contribution to electric permittivity from clusters takes a form:

$\varepsilon_{K}^{\prime}=\frac{P_{0}^{2} L^{3}}{\varepsilon_{0} 2 k_{B} T}\left[\cosh \left(E \frac{P_{0} L^{3}}{2 k_{B} T}\right)\right]^{-2}$

It can be seen in figure 10 that term represented as equation (9) added to equation (5) improves fits and describes experimental data in temperature range from 119 to $153 \mathrm{~K}$. It is interesting to notice that the contribution to electric permittivity from polar clusters at the temperature $121 K$ is about $27 \%$ without dc field (see an example in figure 11) and at the temperature about $130 K$ it is $18 \%$. The contribution from clusters decreases with temperature.

In figure 12 we showed parameters $A$ and $B$ as functions of temperature. The parameter $B$ varies with temperature from $0.5 \cdot 10^{11}$ for higher temperature and rises up to 12 . $10^{11} \mathrm{~m}^{5} \mathrm{VC}-3$ for temperature near the temperature of the phase transition. This increase of $B$ can be connected with existence of the clusters. The parameter $A_{0}$ was found from temperature dependences of parameters $A$. We found that the value of $A_{0}$ is equal to $2.1 \cdot 10^{6} \mathrm{Vm}(\mathrm{CK})^{-1}$, it has the same order as the one calculated from Curie-Weiss $\operatorname{constant}\left(A_{0}=2.8 \cdot 10^{6} \mathrm{Vm}(C K)^{-1}\right)$. The lower value of $A_{0}$ can be connected with the relative volume of cluster, which is comparable with the contribution from clusters to total electric permittivity under zero electric field. In next step according to parameters from formula, which was used for fitting $\varepsilon(E)$ and was obtained by summing equations (4) and (9) we got the clusters size, and polar clusters carried polarization. The following 


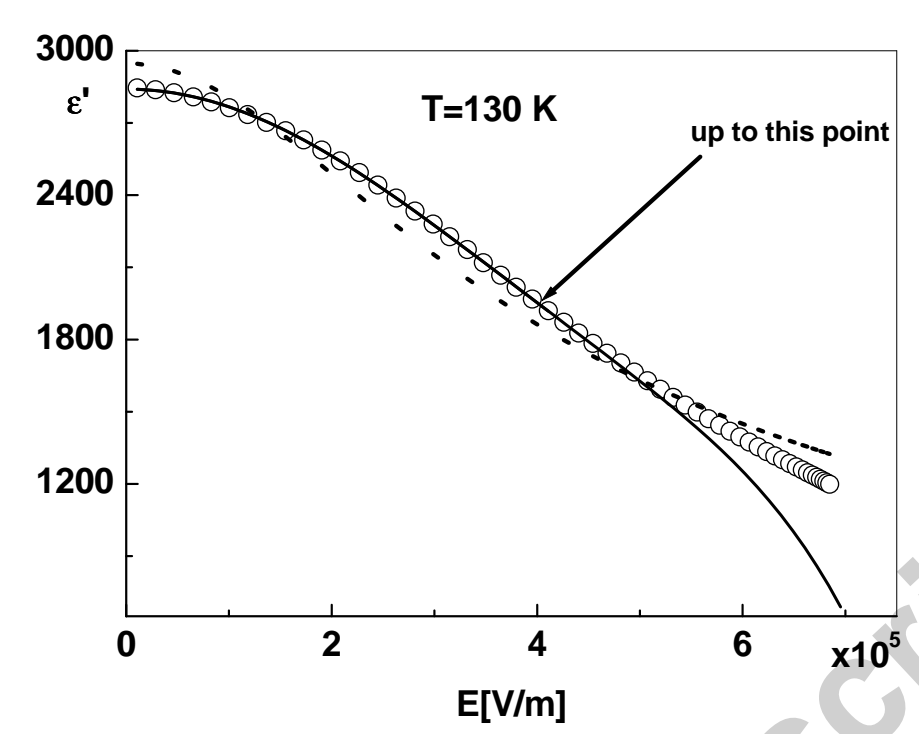

Figure 7. Field dependence of the dielectric constant and its best fit to the equation (5). Dotted line shows fit for an entire range of field, while solid line shows fit for values of field up to $4 \cdot 10^{5} \mathrm{~V} / \mathrm{m}$.

part of equation (9) is connected with clusters. We can rewrite equation (9) in the form:

$\varepsilon_{K}^{\prime}=P_{1}\left[\cosh \left(P_{2} \cdot E\right)\right]^{-2}$,

where parameters $P_{1}$ and $P_{2}$ are:

$P_{1}=\frac{P_{0}^{2} L^{3}}{\varepsilon_{0} 2 k_{B} T}$

$P_{2}=\frac{P_{0} L^{3}}{2 k_{B} T}$

Since $\varepsilon_{0}, k_{B}$ and $\mathrm{T}$ are constant the value of variable $L$ and $P_{0}$ can be calculated from $P_{1}$ and $P_{2}$. We got the clusters size $L=12-20 \mathrm{~nm}$, and polar clusters carried polarization $P_{0}=0.7-4.9 \cdot 10^{-3} \mathrm{Cm}^{2}$. 

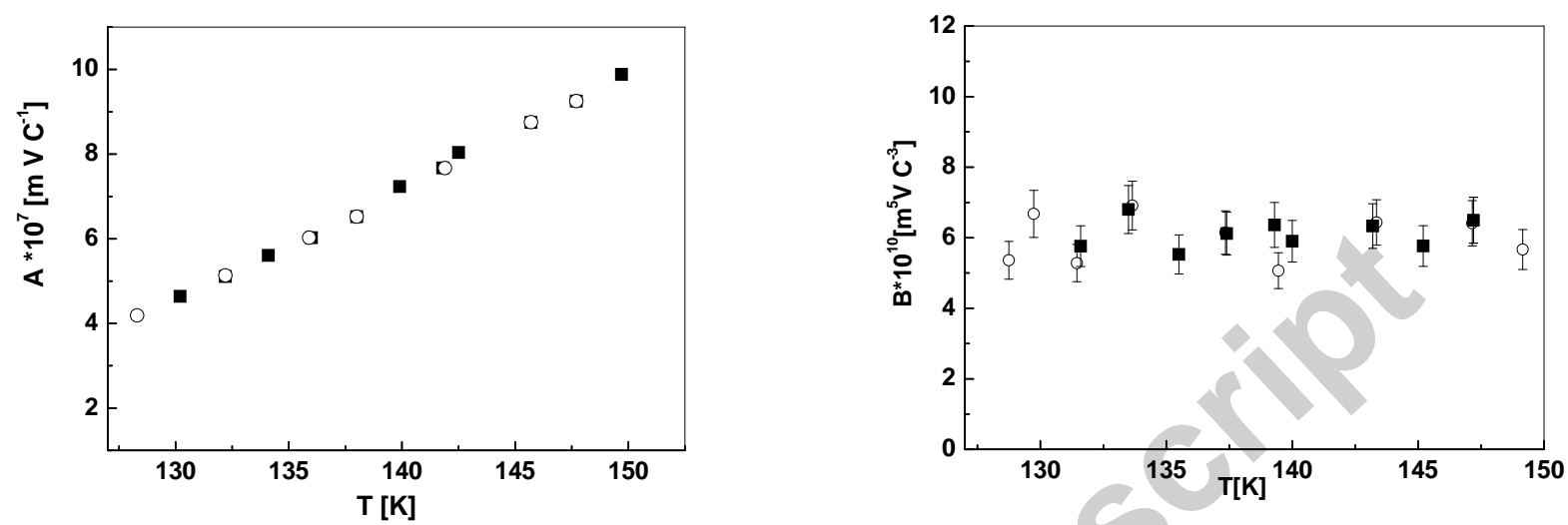

Figure 8. Temperature dependence of fitting parameters $A$ and $B$. In both figures plot with squares denotes dependences according equation (5), while plot with circles denotes dependences according to equation with term $E^{6}$.

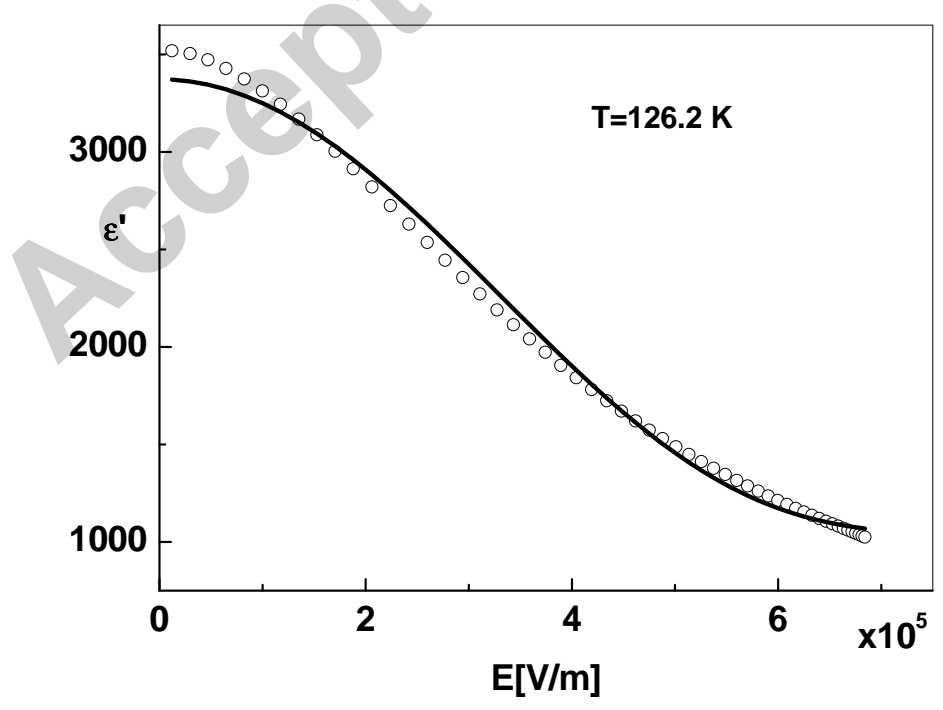

Figure 9. Field dependence of the dielectric constant and its best fit to the equation with $E^{6}$ term (solid line). 


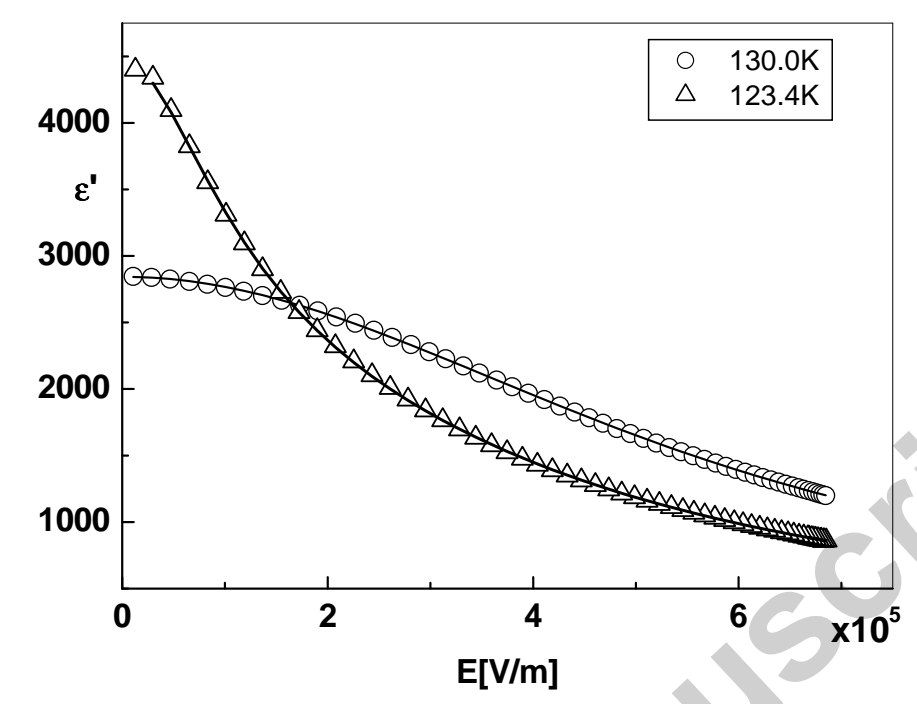

Figure 10. The dc electric field dependence of dielectric constant for two values of temperature. Solid lines represent fits to equation with cluster term.

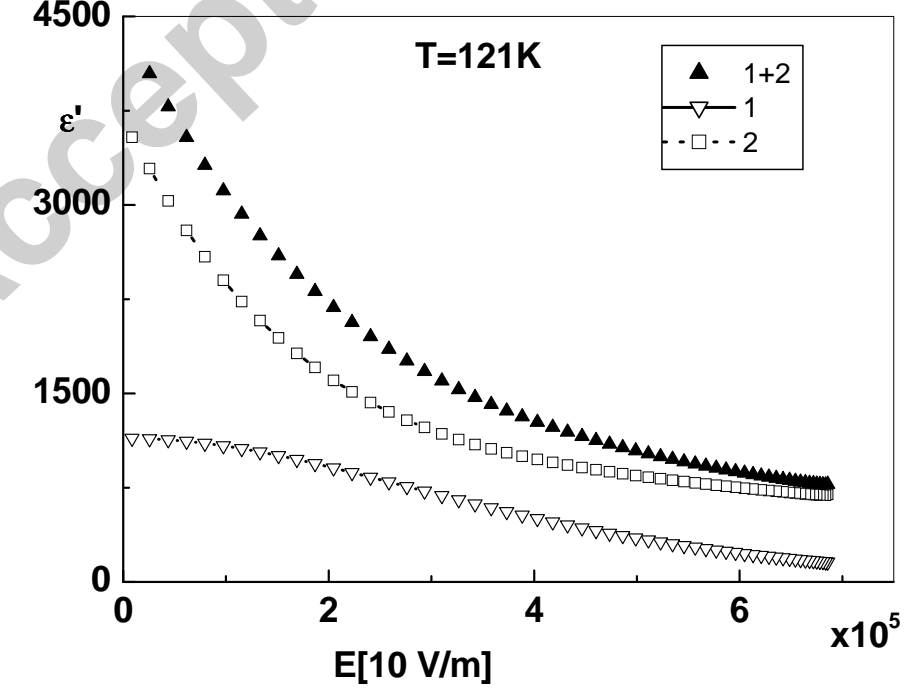

Figure 11. The dc electric field dependence of dielectric constant. Plot labeled as 1 is the contribution from the polar cluster term (according equation (9)), and plot labeled as 2 is the contribution from the conventional polarization term(according equation (5)). 

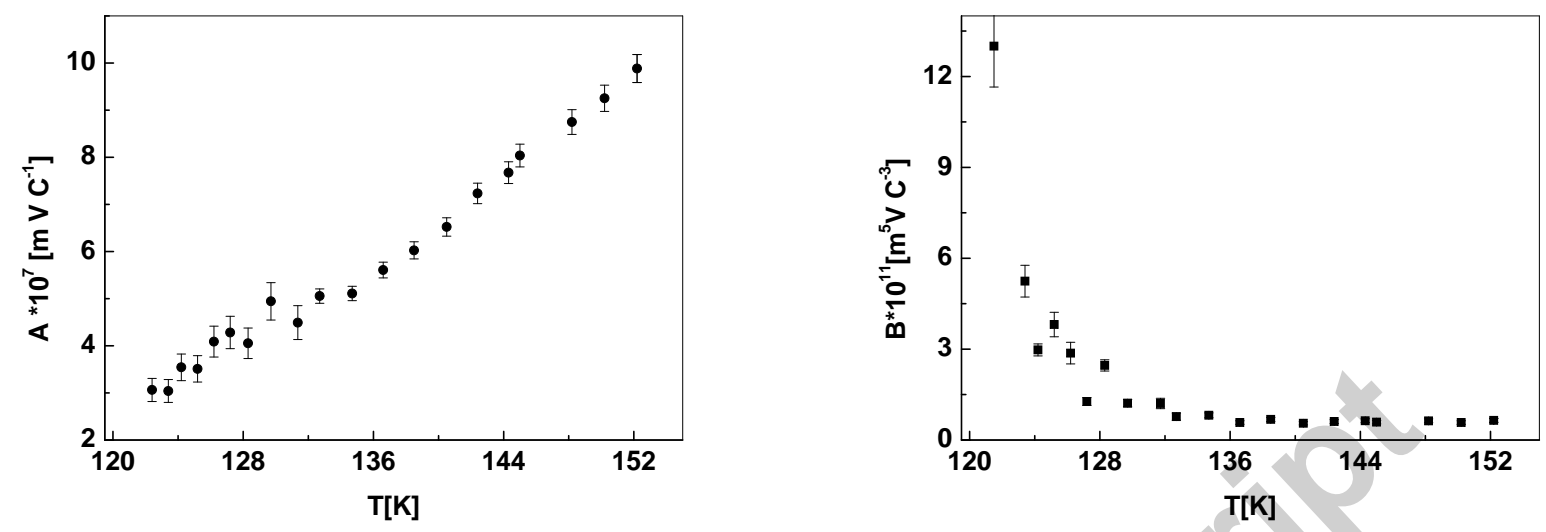

Figure 12. Temperature dependence of parameters $A$ and $B$ of equation with cluster's term.

\subsection{Polarization in the paraelectric phase}

The polarization was measured using an electrometer at frequency of $8.3 \cdot 10^{-3} \mathrm{~Hz}$. Klöpperpier et al. observed that the hysteresis loops in BA exhibit striking S-shaped curves in the interval of a few $K$ above $T_{C 2}$ [1]. In our analysis hysteresis were observed in paraelectric phase even up to temperature $167 \mathrm{~K}$. The correction of polarization obtained by subtracting the part connected with resistance of the sample gives disappearance of hysteresis above $150 \mathrm{~K}$ (see an example figure 13). However, for temperature below $150 \mathrm{~K}$ this method was insufficient to remove hysteresis loops. Character of hysteresis loops after correction is as for ferroelectric one (see figure 13) and one can conclude that presence of these loops confirmed the existence of clusters below $150 \mathrm{~K}$. 


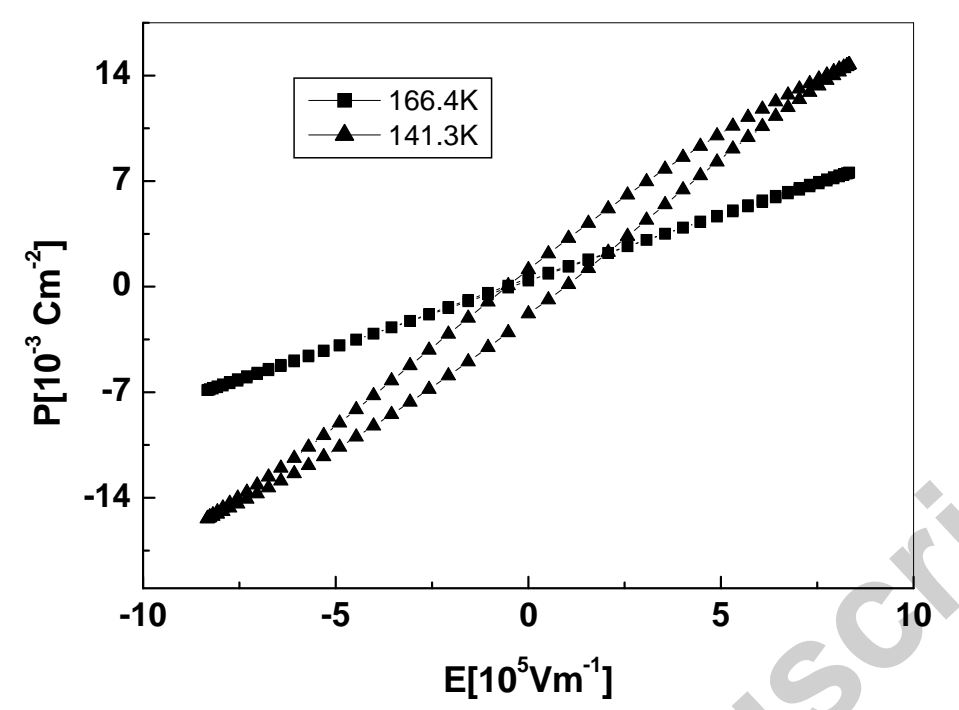

Figure 13. Polarization after correction versus dc electric field.

\section{Summary}

The results obtained in the present study are summarized as follows:

- From relation for $\varepsilon_{\text {max }}^{\prime}(E)$ we found exponent $a=1.66$, which is different than classical one, so the classical equation can not be used in the temperature below $130 K$

- $\varepsilon^{\prime}(E)$ was described using classical state equation with the term $P^{3}$ in the range of temperature $132-152 K$,

- $\varepsilon^{\prime}(E)$ was described using classical relation with term $E^{6}$ in the temperature range $126-152 K$,

- $\varepsilon^{\prime}(E)$ was described by relation with clusters' term added to classical equation in the temperature range $121.5-132 \mathrm{~K}$,

- The existance of clusters with $L=12-20 \mathrm{~nm}$ and $P_{0}=0.7-4.910^{-3} \mathrm{Cm}^{2}$ was confirmed in BA crystal,

- The parameters $A$ and $B$ were obtained by few methods in the broad temperature range; $A_{0}$ is equal to $2.1 \cdot 10^{6} \mathrm{Vm}(\mathrm{CK})^{-1}$ and the parameters $B$ varies with temperature from $0.5 \cdot 10^{11}$ for higher temperature and rises up to $12 \cdot 10^{11} \mathrm{~m}^{5} V C^{-3}$ for temperature near the temperature of the phase transition. 


\section{REFERENCES}

1. Klöpperpieper A, Rother H J, Albers J 1982 Ferroelectrics Letters 44, 115

2. Schildkamp W, Schäfer G, Spilker J 1984 Z. Kristallogr. 168187

3. Rother H J, Albers J, Klöpperpieper A, Müser H E 1985 Jpn. J. Appl. Phys. 24384

4. Freitag O, Brückner H J, Unruh and H G 1985 Z. Phys. B 6115

5. Yuzyuk Y I, Agostinho Moreira J, Almeida A, Chaves M R, Pinto F, Klöpperpieper A 2000 Physical Review B 6115035

6. Frühauf K P, Sauerland E, Helwig J, Müser H E 1984 Ferroelectrics 54293

7. Maeda M, J. Phys. Soc. Jpn. 1988572162

8. Hayase S, Koshiba T, Terauchi H, Maeda M, Suzuki I 1989 Ferroelectrics 96221

9. Almeida A, Carvalho P, Chaves M R, Azevedo, Pires A R, Müser H, Klöpperpier A 1990 Ferroelectrics 108347

10. Agostinho Moreira J, Kiat J M, Chaves M R, Almeida A, Klöpperpieper A 2000 Phys. Stat. Sol. A 178633

11. Almeida A, Carvalho P, Chaves M R, Azevedo J C 1988 Ferroelectr. Lett. Sect. 9107

12. Müser H E, Schell U 1984 Ferroelectrics 55279

13. Schell U and Müser H E 1987 Z. Phys. B 66237

14. Bornarel J, Schmidt V H 1981 J. Phys. C 152017

15. Devonshire A F , 1949 Philos. Mag. 401040

16. Böttcher, Theory of Electric Polarization (Elsevier, Amsterdam, 1977)

17. Dec J, Kleemann W, Itoh M 2005 Phys. Rev. B 71144113

18. Bianchi U, Dec J, Kleemann, Bednorz J G, 1995 Phys.Rev. B 518737

19. Dec J, Kleemann W, Bianchi U, Bednorz J G 1995 Euro. Phys. Letter. 2931

20. Ang Chen, Cross L.E., Guo Ruyan and Bhalla A.S. 2000 Appl. Phys. Lett. 77732

21. Bell A J 1993 J.Phys.Condens.Matter 58773 